\title{
Estado actual de la enseñanza de la filosofía en el nivel educativo medio de Honduras
}

\author{
Ángelo Moreno ${ }^{1}$ \\ Luis Gerardo Reyes Flores ${ }^{2}$
}

\section{RESUMEN}

Esta investigación pretende proporcionar un diagnóstico que permita identificar las dificultades propias de la enseñanza de la filosofía en educación media y delinear algunas posibles soluciones. El estudio se realizó tomando una muestra de estudiantes de 17 instituciones del nivel medio de las cuatro principales ciudades de Honduras y una muestra de sus respectivos docentes. El instrumento englobaba variables como: aspectos relativos a las estrategias didácticas, recursos didácticos, contenidos educativos, rol docente, planeación y evaluación del espacio curricular.

El estudio revela conclusiones que advierten sobre diversos problemas que afronta la enseñanza de la filosofía a nivel de programas, de enfoques pedagógicos y de formación docente, entre otros.

Palabras claves: educación media, enseñanza de la filosofía, estrategias didácticas, recursos didácticos, contenidos educativos.

1 Profesor universitario, beneficiario de una beca básica de la DICYP. Escuela de Filosofía, UNAH: angelomoreno@yahoo.com

2 Profesor universitario, Escuela de Psicología, UNAH: sci.informacion@gmail.com 


\section{ABSTRACT}

This investigation, more than a description, provides a diagnosis that identifies proper difficulties from teaching philosophy in Middle Education and also, outlines some possible solutions. The study was realized, taking as sample students of 17 high schools of the different cities of Honduras and a sample of their respective teachers. The instrument includes variables as: relative aspects of the didactic strategies, educative content, and teachers roll, planning and, evaluation of curricular space.

The study reveals conclusions that warn about different problems in the teaching of philosophy such as pedagogic points of view and teacher formations, among others.

Key words: philosophy, middle education, philosophy teaching, didactic strategies, didactic resources, educative content. 


\section{INTRODUCCIÓN}

En 2012, en la Escuela de Filosofía de la Universidad Nacional Autónoma de Honduras se terminó la investigación: "Percepciones de los estudiantes hacia la clase de filosofía general en el campus central de la Universidad Nacional Autónoma de Honduras"; uno de cuyos objetivos era relacionar las percepciones que habían experimentado los alumnos durante su clase de filosofía en secundaria, con las percepciones de su clase en la universidad.

Una de las conclusiones fue que los alumnos tienen prejuicios negativos acerca de esa asignatura en el nivel medio debido, probablemente, a la falta de prácticas adecuadas por parte de los docentes que imparten las clases; además, que las percepciones de la clase en el nivel superior están fuertemente condicionadas a la experiencia en media. En cambio, las percepciones son favorables, respecto a las experiencias de la clase en la universidad debido a factores subjetivos como el interés personal, la actitud hacia la disciplina de estudio y a la interacción con el docente. Al contrario, estos aspectos se debilitan cuando se analizan las relaciones con los procesos de enseñanza/aprendizaje, con las estrategias y técnicas de planeación y de evaluación, etc.

A partir de ahí surgió la idea de hacer un estudio más profundo que indague sobre las razones que sustentan los datos arrojados en esta investigación. La pregunta subyacente es: ¿cómo inciden las políticas educativas hondureñas (acciones relacionadas con las prácticas educativas y las maneras en que se producen, se distribuyen, se apropian y se expanden las ideas y los conocimientos), en la valoración de la enseñanza de la filosofía en el sistema de educación media?

En Honduras, la enseñanza de la filosofía no está reservada únicamente a los estudiantes que quieren especializarse en esta disciplina o que pretenden hacer de ella una actividad profesional en el futuro. En la tradición hondureña, la clase de la filosofía es de vocación generalista; es decir, además de ser obligatoria, tiene como fin llegar a todos los alumnos de media y desborda el marco estrictamente universitario a nivel de la enseñanza superior. Así, a través del estudio que se ha realizado, se puede afirmar que hay un reconocimiento implícito de que la enseñanza de la filosofía tiene un valor educativo eminente; sin embargo, al parecer, los rasgos distintivos de esta enseñanza no son tan claros. Para la UNESCO (2005, p.2): 
La enseñanza de la filosofía debe mantenerse, defenderse y ampliarse en todo el sistema educativo formal donde ya existe, implantarse donde aún no existe y reponerse allí donde ha sido dejada de lado por diferentes razones, sean estas de carácter utilitario, tecnocrático o economicista. Ahí donde la filosofía se imparte, debe ser nombrada explícitamente con la palabra 'filosofía' y debe estudiarse en unidades académicas autónomas.

Y en otro documento más reciente declara que:

La filosofía encuentra en su enseñanza el ámbito en el que puede desempeñar un papel a la vez esencial y, sin lugar a dudas, arriesgado. Esencial, en la medida en que la enseñanza de la filosofía sigue siendo uno de los elementos clave de la formación para juzgar, criticar, cuestionar y discernir. Arriesgado, puesto que la enseñanza, si se toman en cuenta las mutaciones de una actualidad cada día más rica en historia y en espiritualidad, no se puede pretender atar todos los cabos de lo que puede denominarse «una aceleración el tiempo»: tiempo político, tiempo espiritual, tiempo social y, por tanto, tiempo educativo y pedagógico (UNESCO, 2011, XVII).

En consecuencia, el resultado de la investigación pretende, justamente, determinar las condiciones en las que se encuentra la enseñanza de la filosofía en nuestro país: se trata de un diagnóstico sobre el estado actual de la enseñanza de la filosofía en la educación media en Honduras para obtener datos concretos sobre las características que engloban ese tipo de enseñanza. Los resultados permitirán a los que toman las decisiones sobre las políticas educativas tener un panorama objetivo sobre la situación de esta disciplina y hacer los ajustes y reformas necesarias que estén a tono con las tendencias actuales.

\section{METODOLOGÍA}

Para este estudio se empleó un tipo metodología de corte cuantitativo-descriptivo que permitió hacer uso de las variables que brindan información valiosa para la toma de decisiones.

Se consideró una muestra de 1,709 estudiantes, con un $95 \%$ de confiabilidad doble sigma, teniendo en cuenta una población de 16 instituciones de educación media, distribuidas en las cuatro ciudades con mayor cantidad de habitantes: Tegucigalpa, San Pedro Sula, Ceiba y Choluteca. En el caso de los docentes, se tomó una muestra respectiva al número de instituciones seleccionadas. 
El instrumento para los alumnos se estructuró en ocho apartados, mientras que el de los docentes en cinco. En ambos instrumentos se hizo una medición a través de preguntas cerradas, algunas dicotómicas, otras de selección múltiple; además, se utilizaron las escalas Likert, de valoración y otras que fueron pertinentes para el estudio.

\section{ANÁLISIS Y DISCUSIÓN DE LOS DATOS}

En presente aparatado se presentarán algunos resultados significativos y las correspondientes reflexiones que de estos se pueden realizar. Se estructura en tres subapartados: el primero de ellos hace referencia al tipo de muestra seleccionada y sus características; el segundo, destaca la percepción de los estudiantes con relación a su experiencia en la enseñanza de la filosofía; y el tercero, aborda las experiencias y percepciones de los docentes en cuanto a la enseñanza de la filosofía.

Características de la población encuestada

Del total de la muestra de instituciones, el $53 \%$ está conformado por centros de educación públicos y el $47 \%$ por privados. Igualmente, el porcentaje es proporcional al global de instituciones según su categoría en las 4 principales ciudades del país. La muestra estudiantil (57 \% femenina y $42 \%$ masculina) es consistente, lo que permitió una opinión incluyente en términos de género. La edad promedio de los estudiantes es de 16 años; en su mayoría (45\%) provienen del bachillerato en ciencias y letras, actualmente denominado bachillerato científico humanista en el marco de la nueva reforma educativa en Honduras. El resto del volumen poblacional está distribuido en bachilleratos técnicos profesionales (BTP) y en estudios de formación magisterial.

Percepción de los estudiantes con relación a la clase de filosofía

Las ciencias sociales, en las que se incluye la filosofía, son las menos valoradas; al contrario, las áreas de conocimiento de corte pragmático (ciencias aplicadas y áreas de profesionalización) cuentan con una valoración superior; aun cuando las áreas de conocimiento poseen una valoración que oscila entre 50 y 60 puntos, el área de ciencias sociales figura en las menos valoradas. 
Posicionamiento mental de la filosofía en los estudiantes

En su mayoría (39\%), los estudiantes asocian a la filosofía con el pensamiento racional; no obstante, dicha asociación no es contundente, teniendo en cuenta que el resto de la población asocia la filosofía a aspectos históricos, a la vida y a otros conceptos.

Actividades académicas de mayor frecuencia en el desarrollo de la asignatura

El tipo de aprendizaje que más domina es de corte tradicional. Un $45 \%$ de los estudiantes, tanto de instituciones públicas como de privadas, afirman que se hacen muchas investigaciones, es decir, revisiones bibliográficas; mientras que un $27 \%$ afirma que se elaboran ensayos y se realizan lecturas individuales.

Gráfico 1. Principales fuentes de información utilizadas por los estudiantes en filosofía

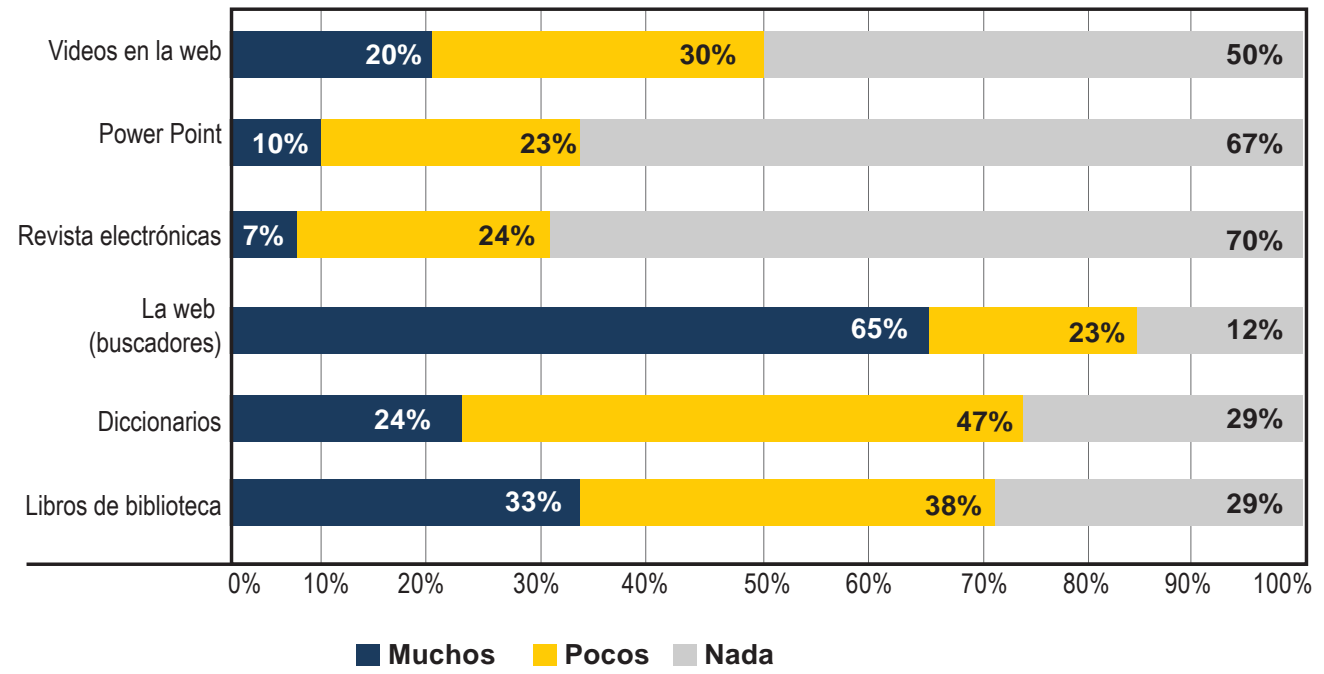

Los jóvenes, en su mayoría, prefieren hacer uso de los medios electrónicos (web); en un nivel medio usan libros de la biblioteca o de diccionarios filosóficos de uso colectivo en las instituciones donde los hay; de igual forma, se aprecia que no existe una cultura de consulta de fuentes especializadas como las bases de datos de revistas indexadas, que son un medio de divulgación de resultados de investigaciones rigurosas (ver gráfico 1).

Los estudiantes perciben que en gran medida se hace uso de estrategias tradicionales o magistrales y, en menor volumen, acciones orientadas al tipo de clase 
constructivista y con base a la adquisición de competencias; posiblemente esto explique la poca valoración por la asignatura (ver gráfico 2).

Gráfico 2. Utilización de recursos didácticos por parte del docente, según estudiantes

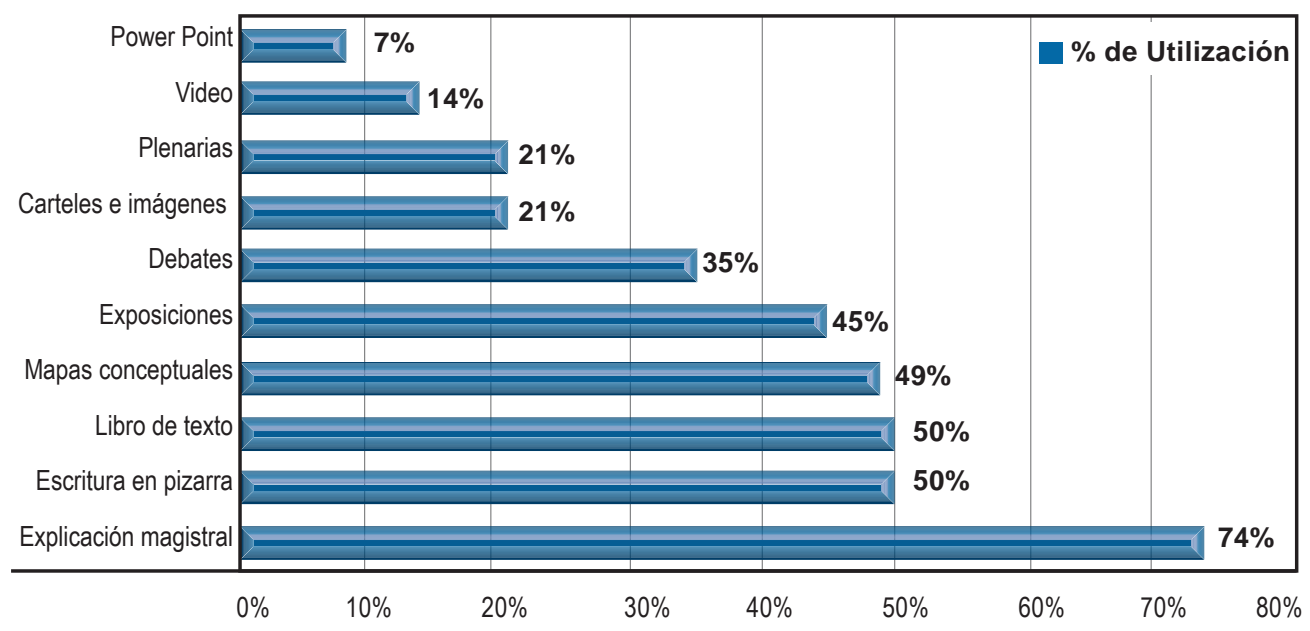

Temas en la enseñanza de la filosofía en el nivel medio de Honduras

El $81.9 \%$ de los estudiantes percibe que se enseña mucho sobre historia de la filosofía, 69.1 \% lógica y 62 \% ética; en menos escala filosofía de la ciencia y filosofía política. Temas como la antropología filosófica, la metafísica, la epistemología y ontología son medianamente o poco enseñados, lo cual indica un posible reduccionismo histórico en la enseñanza de la disciplina en el nivel medio.

Utilidad percibida por los estudiantes de la clase de filosofía en el nivel medio

$78.9 \%$ de los estudiantes consideran que la filosofía está vinculada a la comprensión de los temas religiosos y trascendentales; $74.9 \%$ percibe que la utilidad de filosofía influye en la estructura lógica y en el razonamiento; mientras que un $73.1 \%$ afirma que es útil por el abordaje de temas vinculados a los valores y la moral; no obstante, no fueron valorados en la misma proporción los temas sobre la política y realidad nacional o la evolución del pensamiento científico. Además de lo anterior, se identificó que el $84 \%$ de los estudiantes del nivel medio señala que lo aprendido en la clase de filosofía le será útil en su futuro, ya sea a nivel personal o profesional. 
Experiencias y percepciones de los docentes en cuanto a la enseñanza de la filosofía

1. Formación de los docentes que imparten la clase de filosofía

La mayoría de profesores de filosofía en Honduras cuenta con estudios en ciencias sociales 0 en educación básica; por consiguiente, se deduce que el $60 \%$ de la muestra de docentes cursaron sus estudios en la universidad formadora de docentes, la Universidad Pedagógica Nacional Francisco Morazán; no obstante, al revisar el pensum académico de ambos planes de estudio a nivel de licenciatura, solo se cursan dos espacios curriculares destinados a la filosofía: filosofía general y filosofía educativa. Otro segmento menos representativo de profesores tiene una formación en ciencias religiosas con énfasis en filosofía, se trata de aquellos que realizaron sus estudios en un seminario mayor; en estos casos, un $50 \%$ de la carga académica es de formación en la disciplina filosófica; pero, los estudiantes no se forman en competencias didácticas 0 pedagógicas para la disciplina. En consecuencia, apenas el $14 \%$ de los profesores cuenta con estudios propios de la disciplina y, en su mayoría, han realizado su formación en instituciones extranjeras. Lo anterior permite deducir que, en general, la enseñanza de la filosofía se ve deteriorada por la debilidad en la formación de profesionales de la disciplina. Atendiendo al informe sobre el estado actual del currículo en filosofía para el nivel medio, publicado por la OEI (1998), se observa que esta circunstancia no es exclusiva del contexto hondureño, sino que también es propia en buena parte de Iberoamérica y del mundo.

2. Valoración de referentes para la planificación de la asignatura La mayor parte de docentes (87\%), considera el libro de texto como primer referente primordial; en segundo plano (53\%) el DCNB, cuyas indicaciones sobre la enseñanza de la filosofía son mínimas; y, finalmente, el $33 \%$ de los docentes tiene en cuenta el programa vigente de la Secretaría de Educación de Honduras, el cual data de 1980.

3. Finalidad perseguida por los docentes en la clase de filosofía

El principal interés de los docentes consiste en que los alumnos cultiven la competencia de la argumentación (93\%); siguen en su orden las competencias como la articulación de procesos de problematización y la transferencia de saberes $(73 \%)$ y la capacidad de conceptualización es la que menos interesa (67\%). 


\section{Interés de los docentes por estudiar filosofía}

Se identificó un gran interés por cursar estudios en didáctica de la filosofía (60\%), más que en cursar estudios en filosofía pura; este resultado confirma la sentida necesidad de abrir espacios en esa dirección. Los docentes, en su mayoría, sugieren un tipo de estudios a nivel de maestría o de diplomado de 6 meses.

\section{CONSIDERACIONES FINALES}

En Honduras, la enseñanza de la filosofía en educación media no está reservada únicamente a los estudiantes que quieren especializarse en esta disciplina o que pretenden hacer de ella una actividad profesional en el futuro. En la tradición hondureña, la clase de la filosofía, incluso en la universidad, es de vocación generalista; es decir, tiene como fin llegar a todos los alumnos de media y desborda el marco estrictamente universitario a nivel de la enseñanza superior. A través del estudio que se ha realizado, se puede afirmar que hay un reconocimiento implícito de que la enseñanza de la filosofía tiene un valor educativo eminente, sin embargo, al parecer, los rasgos distintivos de esta enseñanza no son tan claros.

Da la impresión de que la enseñanza de la filosofía es un fin en sí mismo y no un medio para alcanzar varios fines, pues no se trata solamente de abordar o de dar a los alumnos el dominio de un campo disciplinario particular en vista de una eventual especialización en la universidad. A través de la enseñanza de la filosofía lo que se busca es el desarrollo de la libertad de pensamiento, esa libertad es constitutiva en la formación del ciudadano y debe fundar el ideal hondureño de la república "libre, independiente y soberana". Se comprende así que el rasgo de un modelo hondureño debe reposar sobre la unión íntima de una enseñanza escolar y de un régimen político que suponga la existencia de ciudadanos "iluminados", de hombres y mujeres libres.

Así, dado que la enseñanza de la filosofía se sitúa en la educación media y que debe buscar la formación del ciudadano libre, tiene que evitar caer en una formación de erudición y en una reducción enciclopédica; es decir, no se trata de dedicarse a enseñar conceptos aislados de la realidad, ni de enseñar la historia de la filosofía haciendo memorizar nociones y doctrinas de filósofos; más aún, no se trata de adoctrinar sobre temas religiosos o políticos. Al contrario, la enseñanza de la filosofía en educación media debe tener como objetivo primordial construir los primeros elementos de la cultura y del razonamiento en filosofía; en ese sentido, se trata de 
una formación elemental en la que el profesor se debería concebir de manera natural como el "institutor" de sus alumnos en relación a la filosofía; dicho de otra manera, instala al alumno y lo establece dentro del ámbito filosófico. De ahí el rechazo a toda erudición y la resistencia a la implantación de una perspectiva histórica en su enseñanza. Ciertamente, varios elementos históricos son necesarios en una clase de filosofía, sin embargo, cuando se trata de una exposición cronológica de doctrinas muertas o superadas, no vale la pena. Lo que debería buscar esta enseñanza es el desarrollo de la capacidad de reflexión personal del alumno; aunque esto supone el desarrollo de esta misma capacidad por parte del maestro, quien da el ejemplo a través de la construcción de su curso y de la práctica de la lección de filosofía.

El profesor de filosofía no debe estar en situación de exterioridad erudita o histórica en relación a los problemas que trata. Se le pide que se apropie personalmente del contenido de su enseñanza y que manifieste, de manera ejemplar, esa reflexión personal, esa personalización del pensamiento que debe también ser exigida a sus alumnos. Pero, la apropiación personal de la cual el maestro da ejemplo a través de la lección de filosofía, no tiene nada que ver con la sobrevaloración de la subjetividad individual del profesor que hace muestra espectacular de elocuencia frente a sus alumnos. No se trata del enunciado de una opinión personal expresada en primera persona, tampoco es la exposición, incluso brillante, de los sistemas filosóficos. Al contrario, es a través de la búsqueda de lo universal, de la integración de la posición de los problemas por los grandes autores de la tradición filosófica y del rechazo de las opiniones individuales, que se construye progresivamente el curso de la filosofía.

Por tanto, la enseñanza de la filosofía en el sistema de educación media en Honduras debería fundarse sobre la interiorización universalizada de su contenido por el profesor, quien de esta manera está llamado a convertirse verdaderamente en un maestro. Claro está, esta gran ambición no siempre la alcanza el profesor, ni es percibida por los alumnos; sin embargo, ella debe ser el ideal fundador del curso de filosofía. Incluso, si los profesores de filosofía no llegan a convertirse en los autores de su propio curso, deberían, por lo menos, proponer a sus alumnos una progresión y una problematización de las nociones del programa que no pueden simplemente reproducirse a partir de cualquier texto o manual.

Igualmente, la enseñanza de la filosofía en Honduras debería presentarse también como una mezcla única de libertad y de coacción. El profesor es libre de construir su curso, pero al mismo tiempo, debe tratar las nociones del programa. En educación media, el profesor debería poder escoger libremente las obras, pero de una lista de autores que le imponen un límite. El profesor no debe imponer ninguna doctrina ni 
ninguna manera de tratar las cuestiones abordadas, sino que debe evitar la exposición histórica o la simple presentación de las ideas de los filósofos. Además, paradójicamente, el profesor de filosofía no debe estar sujeto a ninguna pedagogía oficial; sin embargo, debe construir la lección de filosofía con los rasgos característicos que han sido señalados antes.

De hecho, la didáctica de la filosofía se engloba dentro de una enseñanza de la libertad que busca el dominio de la expresión organizada de la reflexión personal, a través de la capacidad de articular procesos de problematización (mediante la interrogación), de conceptualización (por medio de la definición de nociones y la expresión de juicios de distinción entre conceptos), de argumentación (a través de la fundamentación y la desconstrucción racional) y finalmente el acto de aplicación y transferencia a la realidad de los resultados significativos obtenidos.

Se trata entonces de un proceso realizado dentro del movimiento y la unidad de un pensamiento ejercido sobre las nociones y las cuestiones fundamentales para todo humano. La finalidad no debe ser, a pesar de las apariencias, el dominio absoluto de la capacidad de argumentar, es decir, de presentar razonamientos convincentes sobre cualquier tema. En filosofía, el razonamiento no puede ser separado de su objeto; no existe ninguna forma vacía de argumentación que pueda atacar a una pregunta desde el exterior; si así fuera, la enseñanza de la filosofía se disolvería en una mera retórica y caería en la sombra de una sofística.

En ese sentido y, a partir de las percepciones presentadas por los mismos maestros, hay que decir que no existe en Honduras un ideal claro de enseñanza de filosofía en educación media. No existen enfoques, programas o premisas claras para guiar la enseñanza en esta disciplina. La enseñanza de la filosofía en educación media en Honduras no tiene rasgos de poseer un verdadero modelo: no parece haber una coherencia interna, ni un carácter sistemático de dependencia recíproca entre los elementos constitutivos de la enseñanza. Esta presupone la inexistencia de un cuerpo de profesores altamente calificados que garanticen su reclutamiento a través de concursos de alto nivel.

Se constata, además, una desvinculación a nivel curricular: no hay programas actuales; el vigente data de 1981. El cuanto al Currículo Nacional Básico, apenas declara que la clase de filosofía hace parte de un área denominada ciencias sociales. En este nuevo modelo del CNB, la educación se estructura en tres ciclos, cada uno de tres años. Se accede a la educación básica, más o menos, a la edad de 6 años y se culmina, aproximadamente, a los 15 (CNB, 2003). 
Durante ese período de aprendizaje, se busca proporcionar a los educandos una educación propicia para la adquisición de elementos básicos de la ciencia, la cultura y la tecnología; además: "Asegura la práctica consciente de los valores esenciales para una convivencia armónica en la sociedad, así como el respeto a los derechos humanos. En este nivel, se busca desarrollar el sentido crítico y autocrítico, el pensamiento reflexivo, racional y lógico y la creatividad" (CNB, 2003, 36).

Un aspecto interesante y que llama la atención, es el hecho de que las áreas curriculares que corresponden a los tres ciclos de este nivel de educación básica son las mismas: comunicación, matemática, ciencias sociales (formación ética y ciudadana) y educación física y deportes. En el documento del CNB $(2003,40)$ se consigna lo siguiente:

El área de Ciencias Sociales comprende los aspectos relacionados con el ser humano, su vida, su organización social en relación con el ambiente y su expresión cultural. Está integrada por diferentes disciplinas tales como Historia, Geografía, Sociología, Demografía, Filosofía, Ética, Política, Economía y otras ciencias. Estas posibilitan el estudio y la comprensión de las sociedades en el tiempo; la diversidad étnica y cultural; la cultura del trabajo y la productividad económica; las formas de organización y la caracterización del cambio social; las relaciones políticas y socioculturales; y la creación de bienes sociales y materiales. Ayuda a comprender el valor social de las instituciones del país, señala las pautas para una convivencia justa y armoniosa, describe las comunidades humanas e identifica las instituciones sociales y sus interacciones con los hombres y mujeres en sociedad. Esta área, igual que las demás, prepara al hondureño y hondureña para desempeñarse con éxito en el mundo globalizado que exige de cada persona una serie de conocimientos, habilidades, actitudes y valores para ser ciudadanos/as productivos/as, creativos/as y competitivos/as.

Así, la filosofía, desde el punto de vista del CNB, pierde la calidad de una clase específica y se disuelve dentro del área de las ciencias sociales. Todos estos elementos deben permitir entrar dentro de una vía serena de reflexión sobre las reformas necesarias e indispensables.

A falta de solidez, la enseñanza de la filosofía en educación media en Honduras corre el riesgo de caer dentro de una fácil demagogia o dentro de un proceso de desconstrucción engañosamente renovadora. En consecuencia, para tratar de asegurar los pilares didácticos de la filosofía en educación media, se hace necesario profundizar sobre la reflexión de la diversidad de situaciones pedagógicas-didácticas que se podrían proponer a los alumnos. 
Esta preocupación por la didáctica no tiene nada de revolucionario, ya que varios países europeos e, incluso, varios latinoamericanos, ya se han lanzado en la investigación sobre las diferentes didácticas de la enseñanza de la filosofía. Sin dejar caer esa reflexión en un "pedagogismo" y, al contrario, sin dejar de darle el paso a la verdadera reflexión didáctica, los profesores de filosofía, respaldados por las autoridades de educación deben comenzar un trabajo grande de reflexión sobre la gran variedad de situaciones que se pueden ofrecer a los alumnos en la clase de filosofía.

En este sentido, una investigación sobre la didáctica de la filosofía en Honduras y sus diferentes formas: normativa, práctica, crítica y prospectiva, se hace necesaria para revisar y evaluar las prácticas mismas. Esto nos ayudaría posteriormente a imaginar todas las actividades, todas las modalidades de trabajo y todos los ejercicios que pueden ser propuestos por los profesores a los alumnos, respetando y consolidando las altas ambiciones culturales e intelectuales de este tipo de enseñanza.

Es en la clase de filosofía que podría superarse, de manera ejemplar, el conflicto entre los que tienen el saber y los pedagogos. Desgraciadamente, este conflicto continúa haciendo un fuerte contrapeso a la evolución indispensable de la enseñanza de la filosofía en educación media.

\section{CONCLUSIONES}

El principal hallazgo del presente estudio derivado del objetivo más importante, radica en que la enseñanza de la filosofía en el nivel medio en Honduras enfrenta severas limitaciones, dado que quienes imparten la asignatura son profesionales de otras áreas de estudio (ciencias sociales, pedagogía, administración educativa); consecuentemente, se conserva un modelo de clase tradicional cuya centralidad se resume en la revisión biográfica e histórica de los filosofía. Este aspecto obedece a la mínima formación relacionada con la disciplina de quienes imparten dicha asignatura; la situación se ve agudizada por la poca producción de profesionales en esta área, aunado a que los filósofos en Honduras no están habilitados para ser parte de la estructura formativa del nivel medio, por aspectos de legalidad en el sistema, lo cual requiere de una revisión del tipo de valoración que se realiza a nivel ministerial, en lo relativo a la enseñanza de la filosofía.

Dentro de las principales acciones pedagógicas que llevan a cabo los docentes se 
aprecia un fuerte uso de recursos y estrategias didácticas de tipo tradicional, en mayor medida el modelo magistral; aún no se han incorporado acciones de carácter constructivista o la formación de competencias en el marco de la asignatura.

En lo relativo a la formación de los docentes, tal como se mencionaba anteriormente, la enseñanza de la disciplina está a cargo de otros profesionales extraños a la disciplina filosófica, lo cual hace que se reste importancia al estudio de la misma y a las bondades de la filosofía como medio que aporte a la construcción social; por consiguiente, el tipo de prácticas de los mismos no corresponden a los nuevos avances en lo relativo a la didáctica de la filosofía; sin embargo, estos profesionales muestran un mediano interés en especializarse en la didáctica de la filosofía, a fin de realizar en buena forma su trabajo profesional.

En cuanto a la valoración de la filosofía, los estudiantes manifiestan un alto grado de valoración, dada la vinculación entre la disciplina y la racionalidad implícita en la misma; aun cuando ellos vinculan esta disciplina a aspectos trascendentales, consideran que es de utilidad en su formación, tanto profesional como actitudinal.

En cuanto a las percepciones según el tipo de administración del centro educativo, no se encontró una diferencia marcada; por lo cual se pude aseverar que la debilidad en la enseñanza de la disciplina no obedece a factores particulares del profesorado, sino que obedece a aspectos curriculares y formativos, derivados del tipo de formación brindada a quienes imparten la asignatura y a la poca o limitada valoración que se le ha dado a la asignatura a nivel ministerial o en lo relativo al diseño curricular, base que gobierna las acciones académicas del nivel medio.

\section{RECOMENDACIONES}

A fin de reivindicar el papel de la filosofía y la utilidad de la misma en el ámbito de la construcción social, se sugiere desarrollar una segunda etapa de investigación que permita identificar el potencial docente a partir de las prácticas didácticas que se desarrollan en el aula de clases, con el objetivo de identificar diversos aspectos valiosos y así echar a andar un plan de formación docente en didáctica de la filosofía; además de ello, se propone consultar a los tomadores de decisiones a nivel educativo para explorar la necesidad de implementar acciones que en otros ámbitos han culminado en experiencias exitosas en torno a la enseñanza de la filosofía. 
Igualmente, se puede crear un escenario que permita la creación de un currículo a nivel de posgrado que dé respuesta a las necesidades formativas en torno a la enseñanza de la filosofía, tales como la didáctica de la misma o un programa a nivel de diplomado que contribuya a la formación de competencias didácticas para la enseñanza de la misma.

Finalmente, como parte de los análisis colaterales del estudio, se plantea la posibilidad de dar continuidad a esta línea de investigación que se ha gestado en el marco de la enseñanza de la filosofía como medio de emancipación de los pueblos, tal como lo propone la UNESCO; por lo tanto, se establece que a mediano plazo se geste la revisión de la enseñanza misma en sus diversos niveles y hacer valoraciones en torno al aporte que puede hacer la filosofía en lo relativo a la formación de ciudadanía y a aspectos axiológicos de suma importancia para el cambio social anhelado desde las raíces mismas de la sociedad.

\section{REFERENCIAS}

Secretaría de Educación. (2003). Currículo Nacional Básico. Tegucigalpa.

UNESCO. (2005). Declaración de Santiago de Chile a favor de la Filosofía, en la conmemoración del Día Mundial de la Filosofía. París: UNESCO.

UNESCO. (2011). Filosofía, una escuela de libertad. París: UNESCO. 